International Journal of Medical Anesthesiology 2021; 4(1): 44-46

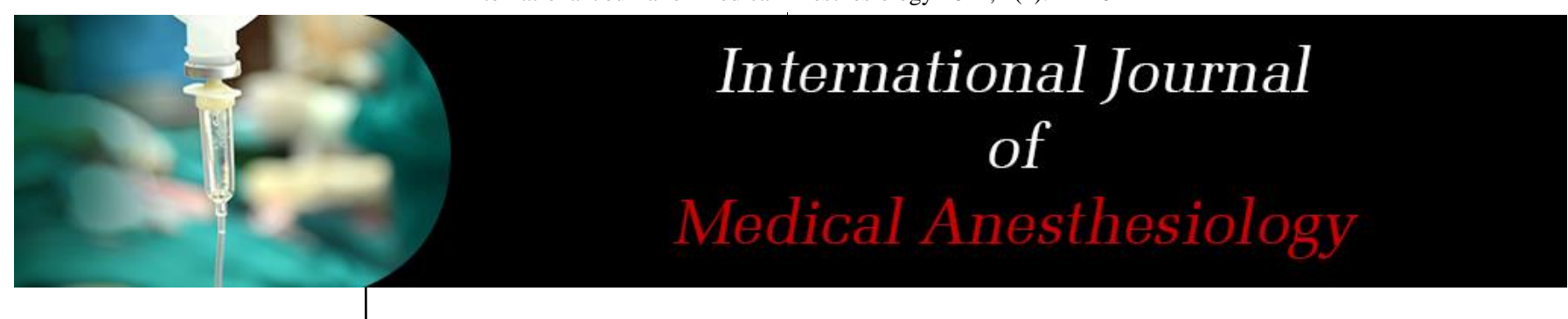

E-ISSN: 2664-3774

P-ISSN: 2664-3766 Www.anesthesiologypaper.com IJMA 2021; 4(1): 44-46 Received: 26-11-2020 Accepted: 29-12-2020

Dr. Joshi Anumeha Assistant Professor, Department of Anesthesiology, Dr. RML Hospital \& ABVIMS Delhi, India

Corresponding Author: Dr. Joshi Anumeha Assistant Professor, Department of Anesthesiology, Dr. RML Hospital \& ABVIMS Delhi, India

\section{Paraquat poisoning: A case report}

\section{Dr. Joshi Anumeha}

DOI: https://doi.org/10.33545/26643766.2021.v4.i1a.195

\section{Abstract}

Paraquat (1, 1' dimethyl 4,4' dipyridylium) is a highly toxic chemical herbicide or week killer. It is one of the most common herbicides used today, but it can cause fatal poisoning when ingested or inhaled. It is often difficult to diagnose in the face of non specific clinical features. Although it is freely available herbicide, few cases have been reported from India. We have reported a case of fatal paraquat poisoning at Hindu Rao Hospital and NDMC Medical College, Delhi.

Keywords: Paraquat poisoning, chemical herbicide, Delhi

\section{Introduction}

Paraquat (1, 1 dimethyl 4,4 dipyridium dichloride) is one of the most widely used herbicides. Name is derived from para positions of the quaternary nitrogens. It is toxic to human beings and animals due to redox activity which produces super oxide anions. Paraquat is metabolized by several enzyme systems viz. NADPH cytochrome P450 reductase, xanthan oxidase, NADH ubiquinone oxidoreductase and NO synthase. In this process, highly reactive oxygen and nitrite species are generated resulting in toxicity in most organs but the toxicity is particularly severe in lungs as paraquat is taken up against a concentration gradient into lungs ${ }^{1}$. It is ingested deliberately or accidentally leading to high mortality due to both local and systemic toxicity ${ }^{[2]}$.

\section{Case report}

A 24-year-old female patient was received in the medical emergency of Hindu Rao Hospital and NDMC medical college, Delhi. There was alleged history of suicidal attempt in which an unknown quantity of a liquid form of poison was consumed by her at her residence. The patient was referred from a local hospital where she was supportively managed with IV fluids. She complained of difficulty in mouth opening, burning in oral cavity and pain on swallowing. There was no history of vomiting, pain abdomen or seizures. On examination, the patient was conscious but confused. Her vitals were found to be normal with heart rate of 88 per minute, BP was $99 / 68 \mathrm{~mm}$ of hg and respiratory rate was 22 . Her temperature was raised to $101.4^{\circ} \mathrm{F}$. Cardiovascular system was normal. There was no difficulty in breathing. No added sounds were present on auscultation of respiratory system. Pupils were bilaterally equal and reactive to light. She was admitted in ICU as a suspect case of organophosphorus poisoning. Tablet charcoal $50 \mathrm{mg}$ was given in bottle of milk of magnesium on day 1 . She was started on injection Pralidoxime $1 \mathrm{mg}$ IV stat on day 1 followed by $500 \mathrm{mg}$ IV TDS was given for 4 days. Injection augmentin 1.2mg IV TDS was given for 3 days. Supportive treatment was given with IV fluids, injection pantoprazole $40 \mathrm{mg} \mathrm{OD}$, emset $4 \mathrm{mg}$ OD and nebulization by asthhalin and budesonide 8 hourly. She was given oxygen therapy by nasal prongs with oxygen at the rate of 3 litres per minute. Her blood and urine reports were normal. She maintained a normal urine output till day 3 . Her vitals were within normal limits till day 3 . Her $x$ ray picture showed bilateral infiltrates on day 3 . Her kidney and liver funtion tests progressively worsened. On the fifth day in ICU, she had erosions in her tongue and oral cavity. (Fig 1) The same day her attendant brought the bottle of the liquid she had consumed (fig 2) and it was paraquat poison. Her clinical picture worsened the same day. She had respiratory rate of 28 per minute and her heart rate was 118 beats per minute. Her chest on auscultation had bilateral crepitations. She was electively ventilated. Her antibiotics were escalated to tazobactam 4.5 gram IV stat followed by 2.25 grams IV TDS and linezolid600mg IV once day. Vitamin C and vitamin E samples were added. 
Injection cyclophosphamide 1 gram IV once daily with 200 $\mathrm{mg}$ normal saline with septran SS injection $400 \mathrm{mg}$ once daily was started. Blood and urine cultured were sterile. Her investigations showed. Her $\mathrm{x}$ ray on day 5 showed features of severe ARDS. She did not respond to the treatment and expired on day 7 post injury as a result of septicemia and respiratory failure.

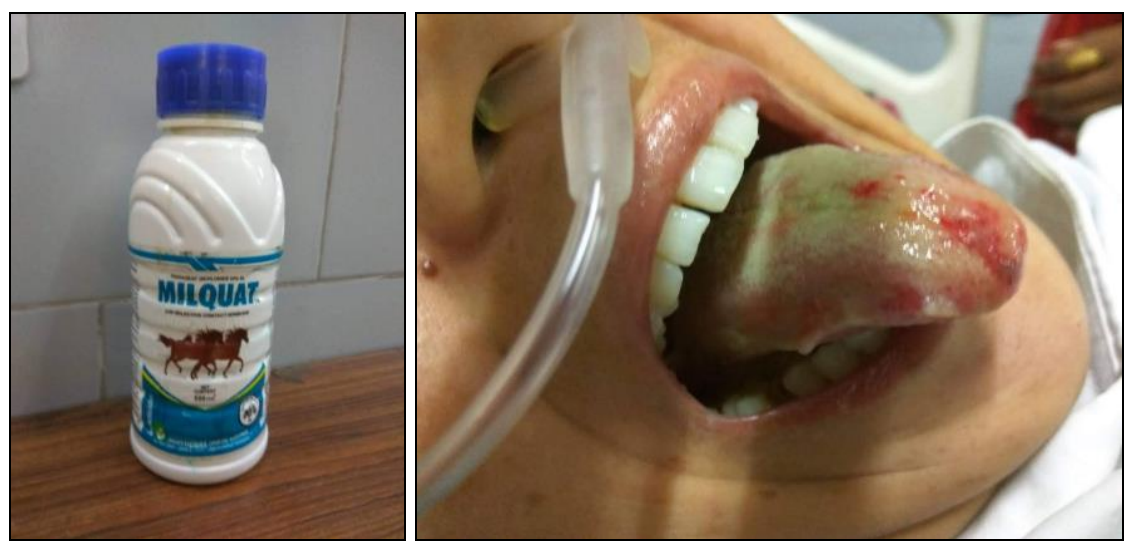

Fig 1-2: Paraquat Tongue fig 2

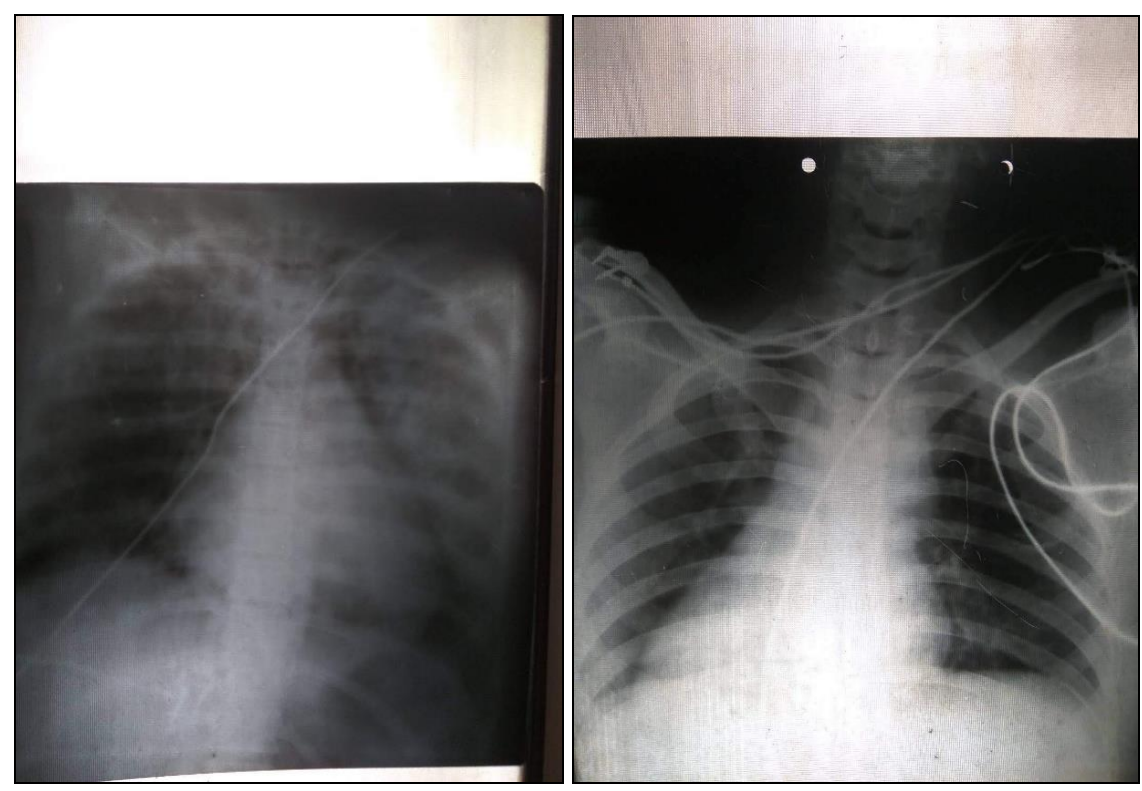

Fig 3-4: $X$ ray picture on day 1

\section{Discussion}

The outcome of paraquat poisoning depends on the severity of the poisoning and the time taken to avail medical help. The high mortality rates are due to the toxicity of the compound itself and the lack of a 5specific antidote. Paraquat toxicity produces local as well as systemic effects $[3,4]$.

Paraquat toxicity is most severe in the lungs ${ }^{[1]}$. Paraquat is actively taken up against a marked concentration gradient into the type II pneumocyte ${ }^{[5]}$. The primary target of toxicity in the lungs is alveolar epithelium. During the acute 'destructive phase' both type I and type II pneumocytes are targetted. Sloughing of the alveoli is associated with pulmonary oedema. This initial phase is followed by a proliferative phase followed by lung fibrosis ${ }^{[6]}$. Kidneys exposed to paraquat demonstrate development of large vacuolation in proximal convoluted tubules which leads to necrosis. Congestion and hepatocellular injury associated with rough and smooth endoplasmic reticulum degranulation and mitochondrial damage occur in the liver. These changes can be observed within a few hours to days.
Upon ingestion, paraquat is rapidly but incompletely absorbed. It is rapidly distributed to lung, liver, kidney and muscle. Paraquat has a volume of distribution of 1.2-1.6 1 $\mathrm{kg}^{-1}$. The initial elimination half-life is around $6 \mathrm{~h}$ but this is around 4 days after the first day. The clinical manifestations that follow paraquat ingestion depend upon the quantity ingested. Ingestion of large amounts of liquid concentrate $(>50-100 \mathrm{ml}$ of $20 \%$ ion $\mathrm{w} / \mathrm{v})$ results in fulminant organ failure ${ }^{[7]}$.

These patients generally have hypoxia, shock and a metabolic acidosis at presentation. Death results from multiple organ failure within several hours to a few days. In our case, the patient had no features of hypoxia or shock at presentation but she rapidly progressed to multi organ failure with liver, kidney nd lung involvement in a span of 7 days. Gastrointestinal toxicity with features of mucosa lesions of mouth and the tongue ('paraquat tongue') as observed in our patient begin to appear within the first few days and may become ulcerated with bleeding.

In congruence to the present case, paraquat ingestion results in an inflammation of the tongue, oral mucosa and throat, 
corrosive injury to the gastrointestinal tract, renal tubular necrosis, hepatic necrosis, and pulmonary fibrosis. ${ }^{8}$ The patient complains of burning and ulceration of the throat, tongue, and esophagus.

Ingestion of smaller quantities usually leads to toxicity in the two key target organs (kidneys and lungs) developing over the next 2-6 days. The patient typically develops increasing signs of respiratory involvement over 3-7 days and ultimately dies of severe anoxia due to rapidly progressive fibrosis up to 5 weeks later. Some liver toxicity is also common in these patients.

Immediate treatment involves removal of ingested paraquat by immediately induced emesis or by gastric lavage in a health care facility. Clay (Fuller's earth) and activated charcoal are effective adsorbents. Administer repeated doses of $60 \mathrm{gm}$ of activated charcoal by gastric tube every two hours for three or four doses. Supplemental oxygen should be withheld unless the $\mathrm{pO}_{2}$ is less than $70 \mathrm{mmHg}$ because oxygen may contribute to the pulmonary damage which is mediated through lipid peroxidation ${ }^{[9]}$.

Hemoperfusion using activated charcoal has been shown to decrease paraquat level, but data to support survival benefit in humans is insufficient. It is only effective if initiated within $4 \mathrm{~h}$ of ingestion, as peak paraquat concentration in the lung is achieved in 5-7 h. Hemodialysis is used as a support of acute renal failure, but it does not increase clearance of the substance as it is rapidly distributed to the lungs and other organs ${ }^{[10]}$. Immunosuppression with combination of cyclophosphamide and methylprednisolone was shown to be beneficial in moderate-to-severe cases by prevention of ongoing inflammation ${ }^{[11]}$.

As there is no specific clinically proven antidote for paraquat poisoning, supportive treatment is given to avoid free radical injury to lungs (vitamins $\mathrm{C}$ and $\mathrm{E}$ ) with pulse therapy using steroids (methylprednisolone or dexamethasone) and cyclophosphamide to prevent pulmonary fibrosis, elimination of paraquat from circulation (hemodialysis), and gastric decontamination. In contrast, the use of oxygen can enhance the toxicity of Paraquat by providing more free radicals ${ }^{[11]}$.

Since the principal biochemical mechanism for lung damage is initiated by oxygen-free radicals produced by peroxidation, clinicians have tried a number of antioxidant treatments in the hope that they might interfere with the process. Unfortunately, none of the studied treatments, including controlled hypoxia, superoxide dismutase, vitamins $\mathrm{C}$ and $\mathrm{E}, \mathrm{N}$-acetylcysteine, desferrioxamine, and nitrous oxide, has been proven to be effective.

\section{Conclusion}

No specific antidote till date is available for paraquat poisoning. Early diagnosis is important for aggressive treatment to prevent further damage. Increased awareness of the clinician and availability of the laboratory diagnostic methods can improve management of paraquat poisoning.

\section{Financial support and sponsorship: Nil}

\section{Conflicts of interest: Nil}

\section{References}

1. Bismuth C, Garnier R, Baud FJ, Muszynski J, Keyes C. Paraquat poisoning. An overview of the current status. Drug Saf 1990;5:243-51.
2. Kondle Raghu, et al. Paraquat poisoning: a case report and review of literature. $\mathrm{J}$ family Community Med 2013;20(3):198-200.

3. Sandhu J, Dhiman A, Mahajan R, Sandhu P. Outcome of paraquat poisoning-a five-year study. Indian $\mathbf{J}$ Nephrol 2003;13:64.

4. Vale JA, Meredith TJ, Buckley BM. Paraquat poisoning: Clinical features and immediate general management. Hum Toxicol 1987;6:41-7.

5. Smith LL, Pratt I, Elliott C, Wyatt I. The accumulation of putrescine and paraquat into lung slices taken from BHT treated mice. Toxicology 1983;27:1-13.

6. Smith P, Heath D. The pathology of the lung in paraquat poisoning. J Clin Pathol Suppl (R Coll Pathol) 1975;9:81-93.

7. Indika B Gawarammana, Nikholas A Buckley. Medical management of Paraquat ingestion. British Journal of Clinical Pharmacology 2011;5:1365-2125.

8. Yang W, Tiffany-Castiglioni E. The bipyridyl herbicide paraquat induces proteasome dysfunction in human neuroblastoma SH-SY5Y cells. J Toxicol Environ Health A 2007;70:1849-57.

9. Olson KR. Poisoning, in Current Medical Diagnosis Treatment, S. J. McPhee, M. A. Papadakis, and L. M. Tierney, Eds., p. 1663, McGraw Hill, New York, NY, USA, 46th edition 2007.

10. Agarwal R, Srinivas R, Aggarwal AN, Gupta D. Experience with paraquat poisoning in a respiratory intensive care unit in North India, Singapore Medical Journal 2006;47(12):1033-1037.

11. Surender Khosya, Sunil Gothwal. Two cases of Paraquat poisoning from Kota, Rajasthan, India. Hindawi. 2012, 3. Article ID 652146 | 